\title{
Formation of Low Voltage Zones on the Anterior Left Atrial Wall Due to Mechanical Compression by the Ascending Aorta
}

\author{
Satoshi Hayashida ${ }^{1}$, Koichi Nagashima ${ }^{1}$, Sayaka Kurokawa ${ }^{1}$, Masaru Arai ${ }^{1}$, Ryuta \\ Watanabe $^{1}$, Yuji Wakamatsu ${ }^{1}$, Naoto Otsuka ${ }^{1}$, Seina Yagyu ${ }^{1}$, Kazuki Iso ${ }^{2}$, and Yasuo \\ Okumura $^{1}$ \\ ${ }^{1}$ Nihon University School of Medicine \\ ${ }^{2}$ Kawaguchi Municipal Medical Center
}

January 27, 2021

\begin{abstract}
BACKGROUND Although low voltage zones (LVZs) in the left atrium (LA) are seen as arrhythmogenic substrate in some patients with atrial fibrillation (AF), pathophysiologic factors responsible for LVZ formation remain unclear. OBJECTIVE To elucidate the anatomical relation between the LA and ascending aorta responsible for remodeling of the anterior LA wall. METHODS We assessed the relation between existence of LVZs on the anterior LA wall and measurements taken on 3dimensional computed tomography images obtained from 102 patients who underwent AF ablation. RESULTS Twenty-nine patients $(28 \%)$ had LVZs $>1.0 \mathrm{~cm} 2$ on the LA wall at the LA-ascending aorta contact area (LVZ Group); no LVZs were seen in the other 73 patients (No LVZ Group). In the LVZ Group (vs. No LVZ Group), the aorta-LA angle was smaller (21.0 $\pm 7.7^{\circ}$ vs. $\left.24.9 \pm 7.1^{\circ}, \mathrm{P}=0.015\right)$, the aorta-left ventricle $(\mathrm{LV})$ angle was greater $\left(131.3 \pm 8.8^{\circ}\right.$ vs. $\left.126.0 \pm 7.9^{\circ} ; \mathrm{P}=0.005\right)$, non-coronary cusp (NCC) diameter was greater $(20.4 \pm 2.2 \mathrm{~mm}$ vs. $19.3 \pm 2.5 \mathrm{~mm} ; \mathrm{P}=0.036)$, and the NCC was closer to the anterior LA wall $(2.29 \pm 0.68 \mathrm{~mm}$ vs. $2.76 \pm 0.79 \mathrm{~mm}$; $\mathrm{P}=0.006)$. The aorta-LA angle correlated positively with patients' body mass index (BMI) and negatively with body weight and BMI. CONCLUSION Deviation of the ascending aorta course and distention of the NCC appear to be related to the development of LA anterior wall LVZs at the LA-ascending aorta contact area. Mechanical pressure exerted by extracardiac structures on the LA along with limited thoracic space may contribute to the development of LVZs associated with AF.
\end{abstract}

Formation of Low Voltage Zones on the Anterior Left Atrial Wall Due to Mechanical Compression by the Ascending Aorta

Satoshi Hayashida, MD,${ }^{1,2}$ Koichi Nagashima, MD, PhD, ${ }^{1}$ Sayaka Kurokawa, MD, PhD, ${ }^{1}$ Masaru Arai, MD, $\mathrm{PhD},{ }^{1}$ Ryuta Watanabe, MD, PhD,${ }^{1}$ Yuji Wakamatsu, MD ${ }^{1}$ Naoto Otsuka, MD ${ }^{1}$ Seina Yagyu, MD,${ }^{1}$ Kazuki Iso, $\mathrm{MD}, \mathrm{PhD},{ }^{2}$ Yasuo Okumura, $\mathrm{MD}, \mathrm{PhD},{ }^{1}$

${ }^{1}$ Division of Cardiology, Department of Medicine, Nihon University School of Medicine, 30-1 Ohyaguchikamicho, Itabashi-ku, Tokyo 173-8610, Japan

${ }^{2}$ Division of Cardiology, Kawaguchi Municipal Medical Center, 180 Nishiaraijyuku, Kawaguchi-shi, Saitama 333-0833, Japan

Address for correspondence:

Koichi Nagashima, MD, PhD; Division of Cardiology, Department of Medicine, Nihon University School of Medicine, 30-1 Ohyaguchi-kamicho, Itabashi-ku, Tokyo 173-8610, Japan

Tel: +81-3-3972-8111 
Fax: +81-3-3972-1098

E-mail: cocakochan@gmail.com

Funding information: none.

Total word count: 2730 words

\section{Brief title: Formation of LVZ on the Anterior LA wall by Mechanical Compression}

The study was supported by departmental resources only, and the authors have no conflict of interest to declare.

\section{Abstract \\ BACKGROUND}

Although low voltage zones (LVZs) in the left atrium (LA) are seen as arrhythmogenic substrate in some patients with atrial fibrillation (AF), pathophysiologic factors responsible for LVZ formation remain unclear.

\section{OBJECTIVE}

To elucidate the anatomical relation between the LA and ascending aorta responsible for remodeling of the anterior LA wall.

\section{METHODS}

We assessed the relation between existence of LVZs on the anterior LA wall and measurements taken on 3-dimensional computed tomography images obtained from 102 patients who underwent AF ablation.

\section{RESULTS}

Twenty-nine patients $(28 \%)$ had LVZs $>1.0 \mathrm{~cm}^{2}$ on the LA wall at the LA-ascending aorta contact area (LVZ Group); no LVZs were seen in the other 73 patients (No LVZ Group). In the LVZ Group (vs. No LVZ Group), the aorta-LA angle was smaller $\left(21.0 \pm 7.7^{\circ}\right.$ vs. $\left.24.9 \pm 7.1^{\circ}, P=0.015\right)$, the aorta-left ventricle (LV) angle was greater $\left(131.3 \pm 8.8^{\circ}\right.$ vs. $\left.126.0 \pm 7.9^{\circ} ; P=0.005\right)$, non-coronary cusp (NCC) diameter was greater $(20.4 \pm 2.2 \mathrm{~mm}$ vs. $19.3 \pm 2.5 \mathrm{~mm} ; P=0.036)$, and the NCC was closer to the anterior LA wall $(2.29 \pm 0.68$ $\mathrm{mm}$ vs. $2.76 \pm 0.79 \mathrm{~mm} ; P=0.006)$. The aorta-LA angle correlated positively with patients' body mass index (BMI) and negatively with body weight and BMI.

\section{CONCLUSION}

Deviation of the ascending aorta course and distention of the NCC appear to be related to the development of LA anterior wall LVZs at the LA-ascending aorta contact area. Mechanical pressure exerted by extracardiac structures on the LA along with limited thoracic space may contribute to the development of LVZs associated with AF.

\section{KEYWORDS}

Ascending aorta, Atrial fibrillation, Extracardiac structures, Left atrial remodeling, Low voltage zones, Voltage mapping

\section{Abbreviations}

AF, Atrial fibrillation; BMI, Body mass index; BW, Body weight; CT, Computed tomography; LA, Left atrium; LCC, Left coronary cusp; LIPV, Left inferior pulmonary vein; LSPV, Left superior pulmonary vein; LV, Left ventricle; LVDd, Left ventricular diastolic diameter; LVDs, Left ventricular systolic diameter; LVEF, Left ventricle ejection fraction; LVZs, Low voltage zones; NCC, Non-coronary cusp; PVI, Pulmonary vein isolation; RCC, Right coronary cusp; RF, Radiofrequency; RIPV, Right inferior pulmonary vein; RSPV, Right superior pulmonary vein; 3D, 3D-dimensional.

\section{Introduction}


Evidence suggests that "atrial fibrillation (AF) begets AF," meaning that AF promotes left atrial (LA) remodeling electrophysiologically and structurally, and vice versa. ${ }^{1}$ Use of a mapping system that allows electrophysiological and anatomical information to be combined has shown that LA remodeling can ultimately manifest as low-voltage zones $(\mathrm{LVZs}) .^{2}$ Previously reported studies have shown existence of LVZs in the LA to be a strong predictor of AF recurrence after pulmonary vein isolation (PVI), ${ }^{3}$ and LVZ ablation added to PVI has been shown to improve ablation outcomes. ${ }^{4}$ LVZs are commonly seen on the anterior LA wall in patients with $\mathrm{AF},{ }^{5-7}$ but pathophysiologic factors responsible for development of such LVZs have not been fully elucidated. Mechanical compression of the LA by extracardiac structures such as the vertebrae and descending aorta has been associated with development of LVZs on the posterior LA wall. ${ }^{5}$ On the basis of this reported association, we hypothesized that mechanical compression by an anatomically deviated or expanded ascending aorta is, at least in part, responsible for development of LVZs on the anterior wall of the LA. We conducted a retrospective study in which we evaluated the relation between anatomical features of the ascending aorta and sinus of Valsalva and distribution of LVZs on the anterior LA wall in patients with AF.

\section{Methods}

\subsection{Study patients}

The study included 102 patients (77 men, 25 women; mean age $64 \pm 10$ years) who had undergone radiofrequency (RF) ablation for AF at Nihon University Itabashi Hospital or Kawaguchi Municipal Medical Center between September 2016 and April 2019. Before the ablation procedure, all had undergone cardiac computed tomography (CT), and all had undergone voltage mapping during sinus rhythm. Transesophageal and transthoracic echocardiography had also been performed before ablation, and the following echocardiographic measures had been obtained; LA diameter, left ventricular diastolic diameter (LVDd) and LV systolic diameter (LVDs), LV ejection fraction (LVEF) (calculated by the Teichholz method), and diastolic function (E/E'). The study was approved by Nihon University Itabashi Hospital Clinical Research Judging Committee and Kawaguchi Municipal Medical Center Ethics Committee.

\subsection{Cardiac imaging and image analysis}

Multi-detector helical 3D-dimensional (3D) CT was performed with a 320-row detector, dynamic volume CT scanner (Aquilion ONE; Toshiba Medical Systems, Tokyo, Japan). The scanning was performed at a slice thickness of $0.5 \mathrm{~mm}$, gantry rotation time of $350 \mathrm{~ms}$, tube voltage of $120 \mathrm{kV}$, and tube current of 300-580 mA for optimum detection of fine structures (resolution of approximately $0.3 \mathrm{~mm}$ ). Electrophysiologic study was performed 18 days after the CT study on average. Each patient's heart rate was maintained at $<65$ bpm by administration of landiolol, and nonionic iodinated contrast (Iomeron, Eisai Co, Tokyo, Japan) was injected at $0.07 \mathrm{~mL} / \mathrm{kg} / \mathrm{sec}$ for 9 seconds. Timing of the image acquisition was determined by bolus tracking software; imaging was initiated when contrast reached the LA. End-expiratory phase images were obtained by gating the image acquisition to $65-75 \%$ of the R-R interval on the lead II electrocardiogram during sinus rhythm or AF rhythm. The acquired CT images were transferred to a workstation (ZIO M900 3.0; QUADRA: Amin Co., Ltd., Tokyo, Japan).

For the purpose of the study, we measured angles between the LA and extracardiac structures in all patients. These were the angle between the midline of the ascending aorta and the midline of the LA (aorta-LA angle), i.e., line connecting the right PV carina to the center of the mitral valve (Figure 1A), and the angle between the midline of the ascending aorta and the left ventricle, i.e., line connecting the mitral valve and LV apex (aorta-LV angle) (Figure 1B). The surface diameter of each aortic valve cusp (LCC, left coronary cusp; NCC, non-coronary cusp; RCC, right coronary cusp), and the minimum distances from the LA to the NCC and LCC were also measured (Figure 1C).

\subsection{Electrophysiologic study with 3D voltage mapping}

All antiarrhythmic drugs had been discontinued for at least 5 half-lives, and the electrophysiologic study with voltage mapping was performed under conscious sedation achieved with dexmedetomidine, propofol, 
and fentanyl. ${ }^{8,9}$ Two long sheaths (an Agilis steerable sheath and an SL0 sheath; St. Jude Medical Inc., St. Paul, MN, USA) were positioned in the LA, and LA pressure was measured. An activated clotting time $>300$ seconds was maintained during the procedure by administration of heparin. The study was performed with use of a 3D mapping system (CARTO 3, Biosense Webster, Diamond Bar, CA, USA) and a multispline catheter with 2-mm interelectrode spacing (Pentaray NAV; Biosense Webster).

High-density electroanatomical maps were obtained during sinus rhythm. If the patient was in AF rhythm, low energy (10-20 joules) intracardiac cardioversion was performed with use of a BeeAT catheter (Japan Lifeline, Tokyo, Japan) placed in the coronary sinus. Bipolar signals were acquired, with a high-pass filter set at $30 \mathrm{~Hz}$ and low-pass filter set at $500 \mathrm{~Hz}$. LVZs were identified as areas with a bipolar peak-to-peak voltage amplitude of $<0.5 \mathrm{mV} .^{4-7,10,11} \mathrm{In}$ addition, the ascending aorta was reconstructed by means of the $3 \mathrm{D}$ mapping system and merged with the LA voltage map. The electroanatomical maps were merged with the CT images that had been obtained.

For the purpose of the study, we measured the LVZs on the anterior LA wall in the areas of contact between the LA and the ascending aorta and sinus of Valsalva.

\subsection{Ablation procedure}

Ablation was guided by the CARTO 3 mapping system, and RF energy was applied by means of a 3.5-mm open-irrigated-tip catheter (Navistar ThermoCoolSmartTouch SF; Biosense Webster) at an irrigation flow rate of $17-30 \mathrm{~mL} / \mathrm{min}$, power of $25-35 \mathrm{~W}$, and temperature of 45 , under sedation achieved by continuous infusion of propofol and dexmedetomidine and intermittent administration of fentanyl. RF was delivered point-by-point with a target contact force of $>10 \mathrm{~g}$, target ablation indices of 450 at the anterior wall and 400 at the posterior wall, and an inter-lesion distance of $<6 \mathrm{~mm}$. A real-time automated tagging module (VisiTag Module, CARTO3; Biosense Webster) was used to assist in the creation of a contiguous PVI circle. The ipsilateral PVs were ablated circumferentially, and successful PVI was affirmed by absence of LA conduction. At least 30 minutes after PVI, $30 \mathrm{mg}$ of adenosine triphosphate was injected to confirm PV conduction block. Electrograms were recorded on a LabSystem PRO (Bard Electrophysiology, Lowell, MA, USA).

\subsection{Follow-up}

Antiarrhythmic drugs were resumed after ablation at the discretion of the attending physician. Twelve-lead electrocardiography was performed for all patients at 1,3, and 6 months, and 24-hour electrocardiographic monitoring was performed every 3 to 6 months. AF occurring 3 months or more after the ablation procedure and lasting at least 30 seconds was diagnosed as AF recurrence.

\subsection{Data analysis}

We divided patients between those with LVZs $>1.0 \mathrm{~cm}^{2}$ on the anterior LA wall at LA-ascending aorta contact areas (LVZ Group) ${ }^{6}$ and those without such LVZs (No LVZ Group). We then compared the following variables between the 2 groups: patients' clinical characteristics, including age, sex, body weight (BW), body mass index (BMI), type of AF (paroxysmal vs. persistent), comorbidities, $\mathrm{CHADS}_{2}$ score, antiarrhythmic drugs used, serum creatinine, NT-proBNP concentrations, post-ablation AF recurrence, echocardiographic variables; LA pressure; and electrophysiologic variables, including the number of voltage mapping points, size (area) of LVZs at LA-ascending aorta contact areas; and CT-based measurements:, including the aortaLA angle, aorta-LV angle, NCC diameter, LCC diameter, RCC diameter, NCC-LA distance, and LCC-LA distance.

\subsection{Statistical analysis}

Continuous variables are expressed as mean +- SD values or median and interquartile range, and categorical variables are expressed as the number and percentage of patients. Student's $t$-test or Mann-Whitney $U$ test was used, as appropriate, to analyze between-group differences in continuous variables, and the chi-square test was used to analyze between-group differences in dichotomous variables unless the expected values in cells were $<5$, in which case Fisher's exact test was used. Strength of the relation between continuous 
variables (size of LVZs at LA-ascending aorta contact areas), BW, BMI, aorta-LA angle, aorta-LV angle, and NCC diameter) was assessed on the basis of Pearson's correlation coefficient. All statistical analyses were performed with JMP 11 software (SAS Institute, Cary, NC), and $P<.05$ was considered statistically significant.

\section{Results}

\subsection{Patient characteristics, echocardiographic variables, and LA pressure}

Of the 102 patients with AF, $29(28 \%)$ had LVZs $>1.0 \mathrm{~cm}^{2}$ on the anterior LA wall at LA-ascending aorta contact areas (LVZ Group), and the other 73 patients had no such LVZs (No LVZ Group). Clinical characteristics of the patients, their echocardiographic variables, and LA pressures are shown per study group in Table 1. Male sex was significantly less prevalent and BW was significantly lower in the LVZ Group than in the No LVZ Group. Maximum LA pressure was significantly greater in the LVZ Group than in the No LVZ Group, and average LA pressure tended to be greater in the LVZ Group. There was no significant between-group difference in comorbidities, prevalence of paroxysmal AF, CHADS2 scores, AF-associated biomarkers, use of antiarrhythmic drugs before the procedure, or echocardiographic variables.

\subsection{Association between anatomical and electrophysiologic features and LVZs}

Electrophysiologic features of the LVZs and CT-based measurements are shown per study group in Table 2. On voltage mapping, 1263 +- 623 points were obtained per patient. In all patients, the NCC and LCC were in contact with the anterior LA wall, and they overlapped the LVZs. A 3D mapping image and anatomical contact between the LA and ascending aorta in a patient with such LVZs is shown in Figure 3. Median size (area) of the LVZs at LA-ascending aorta contact areas was $2.3(1.1-4.0) \mathrm{cm}^{2}$.

The aorta-LA angle was smaller $(21.0+-7.7 \mathrm{deg}$ vs. $24.9+-7.1 \mathrm{deg} ; P=0.015)$, the aorta-LV angle was larger (131.3+-8.8deg vs. $126.0+-7.9 \mathrm{deg} ; P=0.005)$, and the NCC surface diameter was greater $(20.4+-2.2 \mathrm{~mm}$ vs. $19.3+-2.5 \mathrm{~mm} ; P=0.036$ ) in the LVZ Group than in the No LVZ Group (Figure 2A-C).

Results of correlation analysis are shown in Figures 4 and 5. Significant inverse correlation was found between size of the LVZs at LA-ascending aorta contact areas and size of the aorta-LA angle, and significant positive correlation was found between size of these LVZs and size of the aorta-LV angle and of the NCC diameter (Figure 4). Furthermore, NCCs were closer to the anterior LA wall in the LVZ Group than in the No LVZ Group (2.29+-0.68 mm vs. $2.76+-0.79 \mathrm{~mm}$, respectively; $P=0.006)$, but there was no between-group difference in the diameters of the RCC and LCC or in the distance between the LCC and LA (Figure2C). Significant inverse correlation was found between the aorta-LV angle and patients' BW and BMI, and significant positive correlation was found between the aorta-LA angle and patients' BMI (Figure $5)$.

\subsection{Post-ablation AF recurrence}

$\mathrm{AF}$ recurred in 20 patients $(20 \%)$ during a median follow-up period of 16.3 months. The incidence of recurrence was similar between the LVZ Group and No LVZ Group (Table 1).

\section{Discussion}

\subsection{Main findings}

Our main findings were as follows: (1) female sex was more prevalent, BW was lower, and the distance between the NCC and anterior LA wall was less in the LVZ Group than in the No LVZ Group; (2) the size (area) of LVZs on the anterior LA wall at LA-ascending aorta contact areas correlated negatively with the aorta-LA angle and positively with the aorta-LV angle and the NCC diameter; and (3) the aorta-LV angle correlated negatively with BW and BMI, whereas the aorta-LA angle correlated positively with BMI.

In general, LVZs are commonly seen in patients with AF. The incidence among patients with persistent AF is $35 \%$, and among patients with paroxysmal AF, the incidence is $10 \%$. LVZs have been shown to independently predict $\mathrm{AF}$ recurrence after $\mathrm{PVI},{ }^{3,12}$ and LVZs reflecting LA remodeling are frequently detected in the 
anterior LA wall. ${ }^{6,10,11}$ With the ascending aorta being the structure closest to the LA, we hypothesized that mechanical compression caused by an anatomically deviated or expanded ascending aorta leads to the development of LVZs on the anterior LA wall. ${ }^{6}$ The aorta-LA angle was narrow and the aorta-LV angle was wide in our study patients with anterior LA wall LVZs, suggesting that the ascending aorta had sunk into the anterior LA wall due a deviation in its course and distention of the NCC. These deformities might have been the result of aortic root dilation due to aging and hypertension. ${ }^{13,14}$ The NCC is adjacent to the anterior LA wall, and, because of the relatively low LA pressure, it might easily collapse against the LA. Our anatomical findings support our hypothesis. The increased LA pressure in the LVZ Group also supports our hypothesis.

The anterior LA wall LVZs were more prevalent among female patients than among male patients. Generally, the thoracic cavity is smaller in females than in males. Deviation in the course of the ascending aorta and a dilated sinus of Valsalva in patients with a relatively small thoracic cavity might increase intrathoracic pressure. Mechanical compression of the LA by the aorta might promote local myocardial fibrosis and electrophysiologic remodeling. In such cases, the intrathoracic space, which normally compensates for the pressure gradient, would be limited.

We thus suggest that dilation of the aortic root leads to the development of LVZs on the anterior LA wall. LA remodeling is generally thought to be related to the arrhythmic substrate. However, in our study, we found no association between LVZs on the anterior LA wall and AF recurrence after ablation. Remodeling of the anterior LA wall may contribute little to the arrhythmic substrate.

\subsection{Limitations}

Our study was limited by its execution as a retrospective study of a relatively small group of patients. In addition, patient characteristics that might have influenced the study results were not controlled for. Nonetheless, we meticulously evaluated the factors potentially supporting our hypothesis that LVZs on the anterior LA wall develop as a result of a deviated aorta and increased LA pressure. Another limitation is that intracardiac cardioversion was performed in patients who were in AF rhythm before voltage mapping, and LA voltage measured immediately after defibrillation might not be accurate. Further, especially in the patients with persistent AF, the voltage maps obtained during sinus rhythm might not have corresponded precisely to the anatomy depicted by CT performed during AF because of the difference in rhythm and the time between CT study and ablation.

\section{Conclusion}

In examining the relation between anatomical features of the ascending aorta and sinus of Valsalva and distribution of LVZs on the anterior LA wall in patients with AF, we found that a relatively small intrathoracic space along with the relatively low BW and BMI might affect the course of the ascending aorta and lead to mechanical compression of the LA. In addition to this anatomical change, distention of the NCC, increased LA pressure, and sinking of the ascending aorta into the anterior LA appear to promote development of LVZs on the anterior LA wall. Progressive remodeling of the anterior LA wall may not be implicated in LA arrhythmogenicity.

\section{Figure legends}

\section{Figure 1}

Representative 3-dimensional computed tomography images showing how the aorta-left atrium angle (A), aorta-left ventricle angle (B), diameter of coronary cusp, distance from coronary cusp to atrium (C) are measured. Ao = ascending aorta; $\mathrm{LA}=$ left atrium; $\mathrm{LCC}=$ left coronary cusp; $\mathrm{LV}=$ left ventricle; $\mathrm{LVZ}=$ low-voltage zone; $\mathrm{NCC}=$ non-coronary cusp; $\mathrm{RCC}=$ right coronary cusp.

\section{Figure 2}

Representative 3-dimensional computed tomography images showing measurements of the aorta-left atrium angle (A), aorta-left ventricle angle (B), and coronary cusp (C) in patients without LVZs (left panels) and 
patients with LVZs (right panel). Ao = ascending aorta; LA = left atrium; LCC = left coronary cusp; LV $=$ left ventricle; $\mathrm{LVZ}=$ low-voltage zone; $\mathrm{NCC}=$ non-coronary cusp; $\mathrm{RCC}=$ right coronary cusp.

\section{Figure 3}

Representative bipolar voltage map of the left atrium in a patient with LVZs on the anterior LA wall at the LA-ascending aorta contact area. The blue, green, yellow, orange, and red areas indicate LVZs $(<0.5 \mathrm{mV})$, and the purple areas are those of higher voltage $([?] 0.5 \mathrm{mV})$.

LSPV = left atrial pulmonary vein; LVZs = low-voltage zones; RIPV = right inferior pulmonary vein, RSPV $=$ right superior pulmonary vein.

\section{Figure 4}

Scatterplots showing correlation between low-voltage zones (LVZs) $\left(\mathrm{n}=102,0-8.4 \mathrm{~cm}^{2}\right)$ at the LA-ascending aorta contact area and the aorta-LA angle (upper left panel), the aorta-LV angle (upper right panel), and non-coronary cusp (NCC) diameter (lower panel).

$\mathrm{LA}=$ left atrium; $\mathrm{LV}=$ left ventricle.

\section{Figure 5}

Scatterplots showing correlation between patients' body weight (BW) and body mass index (BMI) and the aorta-LA angle (left panels) and the aorta-LV angle (right panels).

$\mathrm{LA}=$ left atrium, $\mathrm{LV}=$ left ventricle.

\section{References}

1. Wijffels MC, Kirchhof CJ, Dorland R, Allessie MA. Atrial fibrillation begets atrial fibrillation. A study in awake chronically instrumented goats. Circulation. 1995;92(7):1954-68.

2. Hohendanner F, Romero I, Blaschke F, Heinzel FR, Pieske B, Boldt LH, et al. Extent and magnitude of low-voltage areas assessed by ultra-high-density electroanatomical mapping correlate with left atrial function. Int J Cardiol. 2018;272:108-12.

3. Verma A, Wazni OM, Marrouche NF, Martin DO, Kilicaslan F, Minor S, et al. Pre-existent left atrial scarring in patients undergoing pulmonary vein antrum isolation: an independent predictor of procedural failure. J Am Coll Cardiol. 2005;45(2):285-92.

4. Rolf S, Kircher S, Arya A, Eitel C, Sommer P, Richter S, et al. Tailored atrial substrate modification based on low-voltage areas in catheter ablation of atrial fibrillation. Circ Arrhythm Electrophysiol. 2014;7(5):82533.

5. Hori Y, Nakahara S, Tsukada N, Nakagawa A, Hayashi A, Komatsu T, et al. The influence of the external structures in atrial fibrillation patients: Relationship to focal low voltage areas in the left atrium. Int J Cardiol. 2015;181:225-31.

6. Nakahara S, Yamaguchi T, Hori Y, Anjo N, Hayashi A, Kobayashi S, et al. Spatial Relation Between Left Atrial Anatomical Contact Areas and Circular Activation in Persistent Atrial Fibrillation. J Cardiovasc Electrophysiol. 2016;27(5):515-23.

7. Nakahara S, Hori Y, Nishiyama N, Okumura Y, Fukuda R, Kobayashi S, et al. Influence of the left atrial contact areas on fixed low-voltage zones during atrial fibrillation and sinus rhythm in persistent atrial fibrillation. J Cardiovasc Electrophysiol. 2017;28(11):1259-68.

8. Wakamatsu Y, Nagashima K, Watanabe I, Watanabe R, Arai M, Otsuka N, et al. The modified ablation index: a novel determinant of acute pulmonary vein reconnections after pulmonary vein isolation. J Interv Card Electrophysiol. 2019;55(3):277-85. 
9. Nagashima K, Watanabe I, Okumura Y, Iso K, Takahashi K, Watanabe R, et al. High-voltage zones within the pulmonary vein antra: Major determinants of acute pulmonary vein reconnections after atrial fibrillation ablation. J Interv Card Electrophysiol. 2017;49(2):137-45.

10. Hori Y, Nakahara S, Nishiyama N, Fukuda R, Ukaji T, Sato H, et al. Impact of low-voltage zones on the left atrial anterior wall on the reduction in the left atrial appendage flow velocity in persistent atrial fibrillation patients. J Interv Card Electrophysiol. 2019;56(3):299-306.

11. Kumagai K, Toyama H, Zhang B. Effects of additional ablation of low-voltage areas after Box isolation for persistent atrial fibrillation. J Arrhythm. 2019;35(2):197-204.

12. Oakes RS, Badger TJ, Kholmovski EG, Akoum N, Burgon NS, Fish EN, et al. Detection and quantification of left atrial structural remodeling with delayed-enhancement magnetic resonance imaging in patients with atrial fibrillation. Circulation. 2009;119(13):1758-67.

13. Boufi M, Guivier-Curien C, Loundou AD, Deplano V, Boiron O, Chaumoitre K, et al. Morphological Analysis of Healthy Aortic Arch. Eur J Vasc Endovasc Surg. 2017;53(5):663-70.

14. Palmieri V, Bella JN, Arnett DK, Roman MJ, Oberman A, Kitzman DW, et al. Aortic root dilatation at sinuses of valsalva and aortic regurgitation in hypertensive and normotensive subjects: The Hypertension Genetic Epidemiology Network Study. Hypertension (Dallas, Tex : 1979). 2001;37(5):1229-35.

\section{Hosted file}

table.pdf available at https://authorea.com/users/349770/articles/506097-formation-of-lowvoltage-zones-on-the-anterior-left-atrial-wall-due-to-mechanical-compression-by-theascending-aorta

\section{Hosted file}

figure.pdf available at https://authorea.com/users/349770/articles/506097-formation-of-lowvoltage-zones-on-the-anterior-left-atrial-wall-due-to-mechanical-compression-by-theascending-aorta 